\title{
The results of introduction study of old garden roses in different natural and climatic zones
}

\author{
Zinaida Klimenko $^{1 *}$, Olena Rubtsova $^{2}$, Svetlana Plugatar $^{1}$, and Vera Zykova ${ }^{1}$ \\ ${ }^{1}$ The Nikita Botanical Gardens - National Scientific Center of the RAS, 298648 Yalta, Russia \\ ${ }^{2}$ M.M.Gryshko National botanical garden NAS of Ukraine, 01014 Kiev, Ukraine
}

\begin{abstract}
The introduction study of old garden roses has been done in the conditions of the forest-steppe zone continental climate ( 69 cultivars) and in xeromorphous edaphoclimatic conditions of the subtropical zone ( 85 cultivars and varieties). The valuable characters of old garden roses in each zone have been determined. The increasing tendency for flower doubleness and their quantity has been educed in a subtropical zone. The long-term introduction study of 21 cultivars in both zones has been done and as the result of it 14 cultivars have been recommended for a wide use in greening.
\end{abstract}

The collections of old garden roses (cultivars of old garden groups, that have been made before 1867) have a great historical and scientific significance: they are the valuable gene fund for a breeding work, and their introduction study allows to take a close look on polymorphy and adaptive capability of Rosa L. genus representatives [1]. For the time being the large collections of old garden roses are collected in N.N.Gryshko National botanical garden NAS of Ukraine, (N.N. Gryshko NBG) and in the Nikita Botanical Gardens - National Scientific Center of the RAS (NBG - NSC) [2-4]. These botanical gardens are located in the zones with different natural and climatic conditions: N.N.Gryshko NBG - in the forest-steppe zone with a moderate continental climate, and NBG - NSC - in the subtropical zone with xeromorphous (Mediterranean type) edaphoclimatic conditions. The work is intended to sum up the long-term introduction study of old garden roses that has been done according to standard methods, in these collections.

The collections of old garden roses of N.N. Gryshko NBG include 69 cultivars of 16 garden groups: Hybrid Perpetual - 16 cultivars (Baron Girod de l'Ain (Reverchon, 1897), Baronne Prevost (Desprez, 1841), Captain Hayward (Bennett, 1893), Empereur de Maroc (Guinoisseau-Flon, 1858), Ferdinand Pichard (Tanne, 1921), Fisher\&Holmes (Verdier, 1865), Frau Karl Druschki (Lambert, 1901), La Reine (Laffay, 1842), Madam Boll (Boll, 1858), Madam Knorr (Verdier, 1855), Marie Baumann (Constantine \& Baumann, 1863), Mrs. John Laing (Bennet, 1885), Paul Neyron (Levet, 1869), Reine de Violettes (MilleMallet, 1860), Sidinie (Vibert, 1847), Souvenir du Docteur Jamain (Lacharme, 1865)); Gallica -8 cultivars (Belle de Crecy (Roeser, 1836), Belle Herminie (Vibert, 1838), Belle Isis (Parmentier, 1845), Cardinal de Richelieu (Parmentier, 1847), Commandamt Beaurepaire (Moreau-Robert, 1864), President de Seze (Hebert, 1828), Versicolor (before

*Corresponding author: klimentina55@mail.ru 
1581), Violacea (before 1795)); Hybrid Rugosa - 6 cultivars (Agnes (Saunders, 1900), Conrad Ferdinand Meyer (Muller, 1893), Hansa (Schaum \&Van Tol, 1905), Nova Zembla (Mees, 1907), Rose a Parfum de l'Hay (Gravereaux, 1901), Tsaritsa Severa (Regel, 1879)); Centifolia - 5 cultivars (Alain Blanchard (Coquerel, 1829), La Rosier Eveque (Descemet, 1815), Madame Hardy (Hardy, 1831), Pompon de Bourogne (before 1664), Village Maid (Vibert, 1839)); Damask and Damask Perpetual - 5 cultivars (Comte de Chambord (Moreau-Robert, 1858), Indigo (Laffay, 1845), Ispahan (before 1827), Jacques Cartier (Desprez, 1842), Leda (1827)); Hybrid Chine - 5 cultivars Comtesse de Woronzoff (Hartwiss, 1828), Duchesse de Montebello (Laffay, 1824), Fortune's Double Yellow (Fortune, 1844), Ruiletii (before 1801), Viridiflora (Smith, 1827)); Bourbon - 5 cultivars (Louise Odier (Margottin, 1851), Madame Isaac Pereire (Garcon, 1881), Madame Pierre Oger (Oger, 1878), Souvenir de la Malmaison (Beluze, 1843), Zephirine Drouhin (Bizot, 1868); Moss - 4 cultivars Chapeau de Napoleon (Kisch, 1827), Madame Louis Leveque (Leveque, 1898), Mousseuse Rouge (before 1842), William Lobb (Laffay, 1855)); Noisette - 3 cultivars (Alister Stella Gray (Grey, 1894), Madame Alfred Carriere (Schwartz, 1875), Marechal Niel (Pradel, 1864)); Alba - 3 cultivars ( Felicite Parmentier (Parmentier, 1836), Madame Plantier (Plantier, 1835), Maiden's Blush (before 1400)); Hybrid Foetida - 3 cultivars (Le Reve (Pernet-Ducher, 1923), Persian Yellow (Willock, 1837), Wildenfels Gelb (Dechant, 1929); Hybrid Spinosissima - 2 cultivars (Hibernica (Templeton, 1802), Stanwell Perpetual (Lee, 1823)); Hybrid Wichurana (Alberic Barbier (Barbier, 1900)); Ayrshire (Duc de Constantine (Soupert\&Notting, 1857)); Hybrid Sempervirens (Felicite et Perpetue (Jacques, 1827)); Hybrid Canina (Kiese (Kiese, 1910)).

85 cultivars and varieties of 20 garden groups are presented in the collection of old garden roses of the NBG - NSC: Hybrid Perpetual - 11 cultivars (Blanck Prince (Paul, 1866), Captain Hayward (Bennett, 1893), Frau Carl Druschki (Lambert, 1901), La Reine (Laffay, 1842), Marie Baumann (Constantine \& Baumann, 1863), Roger Lambelin (Schwartz, 1890), Ulrich Brunner (Levet, 1881), John Hopper (Ward, 1862), Maria Theresa (Ducher, 1872), Mrs John Laing (Bennet, 1885), Paul Neyron (Levet, 1869)); Hybrid Chine -8 cultivars Belle de Nikita (Hartwiss, 1833), Chenedole (Thierry, 1840), Comtesse de Woronzoff (Hartwiss, 1828), Duchesse de Montebello (Laffay, 1824), Fortune's Double Yellow (Fortune, 1844), Gruss an Teplitz (Gerchwind, 1894), Ruiletii (before 1801), Viridiflora (Smith, 1827)); Hybrid Wichurana - 8 cultivars (Alberic Barbier (Barbier, 1900), Albertine (Barbier, 1921), 'American Pillar' (Van Fleet, 1902), Excelsa (Walsh, 1909), Lady Gay (Walsh, 1905), Paul Noel (Tanne, 1910), Pauls Scalet Climber (Paul, 1916), Universal Favorite (Horvath, 1897), White Dorothy Perkins (Cant, 1908)); Gallica 7 cultivars (Belle Isis (Parmentier, 1845), Cardinal de Richelieu (Parmentier, 1847), Charles de Mills (1790), Empress Josephine (Descemet, 1815), Jenny Duval (Duval, 1842), President de Seze (Hebert, 1828), Versicolor (1551)); Hybrid Rugosa - 6 cultivars (Agnes (Saunders, 1900), Blanc Double de Coubert (Cochet,1892), Conrad Ferdinand Meyer (Muller, 1893), F.J. Grootendorst (Goey, 1915), Hansa (Schaum \&Van Tol, 1905), Rose a Parfum de l'Hay (Gravereaux, 1901)); Alba - 6 cultivars (Alba Maxima (before 1500), Celeste (1739), Cocarde (1810), Konigin von Danemark (Booth, 1816), Maiden's Blush (before 1400), Madame Plantier (Plantier, 1835)); Damask and Damask Perpetual - 5 cultivars (Autumn Damask (1849), Comte de Chambord (Moreau-Robert, 1858), Indigo (Laffay, 1845), Ispahan (before 1827), Jacques Cartier (Desprez, 1842), Petite Lisette (Vibert, 1817), Pink Leda (before 1827)); Moss - 5 cultivars ((Felicite Parmentier (Parmentier, 1836), Goethe (Lambert, 1911), Henri Martin (Laffay, 1862), Nuits de Young (Laffay, 1845), Violacee (Soupert \& Notting, 1876)); Noisette - 4 cultivars (Blush Noisette (1817), Gloire de Dijon (Jacotot, 1853), Madame Alfred Carriere (Schwartz, 1875), Marechal Niel (Pradel, 1864)); Bourbon - 4 cultivars (Hermosa (Marchesseau, 1832), Louise Odier (Margottin, 1851), Madame Isaac Pereire (Garcon, 1881), Madame Pierre 
Oger (Oger, 1878)); Centifolia - 2 cultivars (Alain Blanchard (Coquerel, 1829) and Minette (Vibert, 1819)); Bengale - 2 cultivars Mutabilis (before 1894) and Old Blush (1793)); and other garden groups with occasional: Ayrshire (Duc de Constantine (Soupert\&Notting, 1857)), Hybrid Sempervirens (Felicite et Perpetue (Jacques, 1827)), Tea (Marie Van Houtte (Ducher, 1871)), Hybrid Foetida (Persian Yellow (Willock, 1837)), Hybrid Musk (Prosperity (Pemberton, 1919)), Hybrid Spinosissima (Stanwell Perpetual (Lee, 1821)), Hybrid Multiflora (Wartburg (Kiese, 1910)), Polyantha (Yvonne Rabier (Turbat, 1910)). The collection also has 6 historical rose varieties and forms, which were introduced in Europe before the beginning of XXth century: Rosa banksiae Aiton Alba (1807), Rosa banksiae Aiton Lutea (1824), Rosa bracteata Wendland. (1793), Rosa chinensis semperflorens (Curt.) Koehne (before 1658), Rosa fortuneana Lem. (1850), Rosa indica odoratissima Lindley (1808).

It can thus be seen that in the conditions of a subtropical climate the introduction testing of heat-loving climbing roses from groups Hybrid Chine, Hybrid Wichurana as well as climbing varieties and forms is widely done. The historical traditions in the landscape design in the Southern Coast of the Crimea happened to use old garden roses Alberic Barbier, Albertine, American Pillar, Comtesse Elisabeth Woronzow, Dorothy Perkins, Duc de Constantin, Félicité et Perpétue, Fortune's Double Yellow, Maréchal Niel, Paul's Scarlet Climber, White Dorothy Perkins, Excelsa, as well as varieties and forms Rosa banksiae Alba, Rosa banksiae Lutea, Rosa bracteata, Rosa fortuneana [5] in different types of a vertical greening. The valuable character of many old garden roses in the conditions of a subtropical climate is very early initial blossom. So Rosa banksiae Alba, Rosa banksiae Lutea and Rosa fortuneana initiate blossom in the second decade of April. Rosa indica odoratissima, Alberic Barbier, Comtesse Elisabeth Woronzow, Duc de Constantin, Fortune's Double Yellow, Marie Van Houtte, Pauls Scalet Climber, Persian Yellow, Ruiletii are in the list of early starting to bloom. They also have remarkably abundant flowering, and it creates a high decorative effect.

In the conditions of a moderate continental climate old garden roses are withstanding enough. 40 old garden roses' cultivars from the quantity of the studied ones proved to be winter-hardy. And 17 cultivars (Alain Blanchard, Alberic Barbier, Baron Girod de l'Ain, Captain Hayward, Felicite et Perpetue, Felicite Parmentier, Jacques Cartier, Le Rosier Eveque, Louise Odier, Madame Alfred Carriere, Maiden's Blush, Marechal Niel, Reine de Violettes, Souvenir de Docteur Jamain, Versilocor, Violacea, Viridiflora) are shade enduring plants.

As a whole, the introduction study showed that many old garden roses' cultivars are resistant to fungus deceases. Among their values are high decorative qualities of a flower and as a rule a frank pleasant fragrance.

The long-term introduction study of 21 cultivars in both zones has been done. It has been discovered that as a general matter the morphological and biological features of the cultivars are stable in both zones during cultivation. However, in the process of comparison of morphometrical characteristics it was discovered that the increasing tendency for flower doubleness and their quantity had been educed in a subtropical zone. It is represented in Table 1.

As the result of cultivars' perspective assessment for use in each climatic zones, for a forest-steppe zone 14 cultivars are recognized as prospective ones and for a subtropical zone -19 cultivars. 14 cultivars are recommended for a wide use in both zones' greening. These highly decorative cultivars with scented flowers are Agnes, Duc de Constantine, Frau Karl Druschki, Fortune's Double Yellow, Jacques Cartier, La Reine, Louise Odier, Madame Alfred Carreire, Madame Hardy, Maiden's Blush, Marie Baumann, Persian Yellow, President de Seze, Rouletii. 
Table 1. Some results of comparative assessment of old garden roses' cultivars in the collections of N.N.Gryshko NBG and NBG - NSC.

\begin{tabular}{|c|c|c|c|c|}
\hline Cultivar & $\begin{array}{c}\text { Maximum } \\
\text { flower diameter } \\
\text { N.N. Gryshko } \\
\text { NBG /NBG- } \\
\text { NSC, cm }\end{array}$ & $\begin{array}{l}\text { Maximum petal } \\
\text { quantity } \\
\text { N.N. Gryshko } \\
\text { NBG /NBG- } \\
\text { NSC, pes }\end{array}$ & $\begin{array}{l}\text { Maximum flower } \\
\text { quantity in a } \\
\text { flower head N.N. } \\
\text { Gryshko NBG } \\
\text { /NBG-NSC }\end{array}$ & $\begin{array}{c}\text { Shrub } \\
\text { highness } \\
\text { (scion } \\
\text { length), } \\
\text { cm }\end{array}$ \\
\hline Agnes & $7 / 10$ & $85 / 80$ & $3 / 5$ & $2.5 / 2.5$ \\
\hline $\begin{array}{l}\text { Alberic } \\
\text { Barbier }\end{array}$ & $7 / 8$ & $16 / 83$ & $1 / 13$ & $2.5 / 5$ \\
\hline $\begin{array}{c}\text { Cardinal de } \\
\text { Richelieu }\end{array}$ & $6 / 7$ & $50 / 60$ & $3 / 3$ & $1.5 / 1.7$ \\
\hline $\begin{array}{c}\text { Comte de } \\
\text { Chambord }\end{array}$ & $12 / 10$ & $55 / 55$ & $5 / 5$ & $1.5 / 1.5$ \\
\hline $\begin{array}{l}\text { Comtesse de } \\
\text { Woronzoff }\end{array}$ & $10 / 10$ & $40 / 40$ & $5 / 5$ & $3 / 3$ \\
\hline $\begin{array}{c}\text { Duc de } \\
\text { Constantine }\end{array}$ & $9 / 9$ & $40 / 75$ & $5 / 6$ & $2 / 7$ \\
\hline $\begin{array}{l}\text { Felicite et } \\
\text { Perpetue }\end{array}$ & $5 / 4$ & $45 / 60$ & $10 / 10$ & $2.5 / 5$ \\
\hline $\begin{array}{l}\text { Frau Karl } \\
\text { Druschki }\end{array}$ & $14 / 14$ & $36 / 35$ & $3 / 5$ & $2 / 1.5$ \\
\hline $\begin{array}{c}\text { Fortune's } \\
\text { Double } \\
\text { Yellow } \\
\end{array}$ & $9 / 12$ & $16 / 32$ & $3 / 3$ & $1.5 / 4$ \\
\hline Indigo & $8 / 8$ & $60 / 35$ & $3 / 3$ & $1.2 / 2$ \\
\hline $\begin{array}{l}\text { Jacques } \\
\text { Cartier }\end{array}$ & $7 / 9$ & $70 / 75$ & $5 / 5$ & $2 / 1$ \\
\hline La Reine & $12 / 10$ & $78 / 100$ & $3 / 3$ & $1.7 / 1.2$ \\
\hline $\begin{array}{c}\text { Louise } \\
\text { Odier }\end{array}$ & $9 / 8$ & $56 / 65$ & $5 / 3$ & $1.8 / 2$ \\
\hline $\begin{array}{l}\text { Madame } \\
\text { Alfred } \\
\text { Carreire }\end{array}$ & $10 / 11$ & $30 / 45$ & $5 / 5$ & $2.5 / 2.5$ \\
\hline $\begin{array}{c}\text { Madame } \\
\text { Hardy }\end{array}$ & $8 / 9$ & $25 / 55$ & $5 / 5$ & $2 / 1,5$ \\
\hline $\begin{array}{l}\text { Maiden's } \\
\text { Blush }\end{array}$ & $9 / 10$ & $80 / 80$ & $5 / 5$ & $2 / 3$ \\
\hline $\begin{array}{c}\text { Marechal } \\
\text { Niel }\end{array}$ & $11 / 11$ & $30 / 30$ & $3 / 6$ & $2 / 6$ \\
\hline $\begin{array}{c}\text { Marie } \\
\text { Baumann }\end{array}$ & $9 / 10$ & $75 / 80$ & $5 / 5$ & $2 / 2,3$ \\
\hline $\begin{array}{l}\text { Persian } \\
\text { Yellow } \\
\end{array}$ & $7 / 6$ & $40 / 40$ & $1 / 6$ & $2 / 2$ \\
\hline $\begin{array}{c}\text { President de } \\
\text { Seze }\end{array}$ & $7 / 9$ & $75 / 80$ & $7 / 8$ & $1.5 / 1.6$ \\
\hline Rouletii & $4 / 4$ & $40 / 28$ & $15 / 26$ & $0.6 / 0.5$ \\
\hline
\end{tabular}

Thus, as the result of the study, promising use of old garden roses in modern landscape design and in a breeding work has been discovered. The assortment, that is prospective for 
cultivation in each zone, as well as 14 cultivars, that are recommended for both a foreststeppe zone and a subtropical zone, has also been discovered.

\section{References}

1. Z. Klimenko, O. Rubtsova, V. Zykova, Plant Introduction 3, 49-52 (2006)

2. Z. Klimenko, N. Zubkova,V. Zykova, S. Pluhatar, I. Kravchenko, E. Karpova, A. Shvets, Annotated catalog of ornamental plants of the Nikitsky Botanical Gardens. Volume 1. Collections of garden roses, clematis, lilac (ph "Arial", Simferopol, 2018)

3. O. Rubtsova, V. Chizhankova, Plant Varieties Studying and Protection 2, 5-9 (2016)

4. O. Rubtsova, V. Chizhankova, Old roses (Kiev, 2019)

5. Yu. Plugatar, Z. Klimenko, S. Plugatar, V. Zykova, I. Kravchenko, Acta Hort. 1201, 655-658 (2018) 